Etnográfica

Revista do Centro em Rede de Investigação em

Antropologia

vol. $23(3)$ | 2019

Vol. 23 (3)

\title{
Formarse al interior de un linaje: el caso de los estudiantes de teatro del circuito realista en
}

\section{Buenos Aires}

Educated within a lineage: the case of theater students of the realist circuit in

Buenos Aires

\section{Santiago Battezzati}

\section{(2) OpenEdition}

\section{Journals}

Edición electrónica

URL: https://journals.openedition.org/etnografica/7544

DOI: 10.4000/etnografica.7544

ISSN: 2182-2891

\section{Editor}

Centro em Rede de Investigação em Antropologia

Edición impresa

Fecha de publicación: 1 octubre 2019

Paginación: 739-752

ISSN: 0873-6561

Referencia electrónica

Santiago Battezzati, «Formarse al interior de un linaje: el caso de los estudiantes de teatro del circuito realista en Buenos Aires», Etnográfica [En línea], vol. 23 (3) | 2019, Publicado el 28 noviembre 2019, consultado el 20 enero 2022. URL: http://journals.openedition.org/etnografica/7544 ; DOI: https:// doi.org/10.4000/etnografica.7544

\section{(c) (7) (8)}

Etnográfica is licensed under a Creative Commons Attribution-NonCommercial 4.0 International License. 


\section{Formarse al interior de un linaje: el caso de los estudiantes de teatro del circuito realista en Buenos Aires}

\section{Santiago Battezzati}

Recientemente, algunos estudios de antropología urbana se han interesado por varias prácticas que se aprenden y/o se realizan en circulación. Asimismo, algunos trabajos han llamado la atención sobre trayectorias o circuitos particulares. Basado en una investigación etnográfica, este trabajo busca analizar un tipo particular de trayectorias entre algunos estudiantes de actuación en Buenos Aires. Como en otras actividades, los estudiantes de teatro en Buenos Aires se forman circulando por diferentes estudios. Sostendremos aquí que, para el caso del circuito realista, esta circulación no se efectúa simplemente entre maestros que enseñan en un mismo estilo o estética, sino entre maestros que pertenecen a un mismo linaje, y que dicho fenómeno debe entenderse en el contexto de una cierta memoria colectiva en la que los actores que estudian con maestros de un cierto linaje se van socializando.

PALABRAS-CLAVE: circuito, teatro, performance, linaje, memoria colectiva.

Educated within a lineage: the case of theater students of the realist circuit in Buenos Aires - Recently, many urban anthropologists have described how people learn some corporal practices by moving along the city. Also, some studies have underlined the existence of specific circuits. Based on an ethnographic research, this paper will analyze a specific circuit between theater students in the city of Buenos Aires. I will show that, for the "realist" circuit, students choose their teachers not only with esthetic criteria or depending on the teaching method. Instead, they look for teachers that belong to a particular lineage. This can be explained in the context of a certain collective memory in which students are socialized while learning theater with teachers of a certain lineage.

KEYWORDS: circuit, theater, performance, lineage, collective memory.

BATTEZZATI, Santiago (santiagobattezzati@gmail.com) - Conicet/IDAES, Universidad de San Martín, Argentina. 


\section{INTRODUCCIÓN}

El aprendizaje y práctica de disciplinas en el contexto urbano se suelen dar en diferentes lugares, espacios públicos y/o establecimientos privados a lo largo de la ciudad. ${ }^{1}$ ¿Cuáles son las formas específicas que toma esta circulación? En los últimos años, algunos estudios en antropología urbana se han interesado por este fenómeno. Tal es el caso de Magnani (1999) para el circuito neoesotérico en San Pablo, Carozzi (2000) para el circuito alternativo y Osswald (2015) para la danza independiente en Buenos Aires, Del Mármol (2016) para el aprendizaje de teatro y Sáez (2017) para la danza contemporánea y el circo en La Plata. En la mayoría de los casos, los límites de esa circulación parecen coincidir con la totalidad de los lugares que ofrecen un servicio determinado en una ciudad o territorio delimitado.

Paralelamente, algunos estudios se han encargado de mostrar la existencia de modos diversos - y a veces enfrentados - de concebir la práctica de una disciplina que organizan fronteras simbólicas al interior de la misma. Particularmente interesante es el trabajo de Infantino (2014), quien analiza las disputas identitárias e intergeneracionales en la definición de una identidad de la comunidad circense en Buenos Aires, que esta antropóloga estudia como una formación cultural. Infantino muestra el modo en que algunas personas marcan diferencias sobre las formas de aprender, enseñar y hacer circo, principalmente en las oposiciones que se generan entre nuevo circo, circo callejero y circo tradicional. Por su parte, Carozzi (2015) también señala un conflicto intergeneracional para el caso del baile del tango, que tiene su origen en diferentes expectativas cinético morales de bailarines habitués de milongas ortodoxas y milongas relajadas. En ambos casos, las diferenciaciones y las fronteras que se establecen se encuentran, en buena medida, en la forma en que los otros se mueven y realizan la práctica. El principal interés de estos trabajos para el problema de la circulación radica en que, al señalar la existencia de fronteras - en lo relativo a ciertas formas de moverse y usar el cuerpo -, plantea, al menos como posibilidad, la existencia de diferentes circulaciones al interior de una misma disciplina. Esta cuestión es fundamental porque, si la circulación como fenómeno ha sido mencionada por varios estudios, menos han sido los trabajos que se han encargado de los límites o la delimitación de la circulación. ${ }^{2}$

l Este artículo forma parte de una investigación etnográfica centrada en la formación de actores de teatro en dos estilos de actuación en Buenos Aires. La mayor parte del trabajo de campo se realizó entre 2014 y 2016, y consistió en un trabajo etnográfico en clases de actuación, así como otras actividades realizadas con estudiantes de actuación (como asistir a varietés y obras de teatro con ellos, entre otras). También se realizaron entrevistas en profundidad con estudiantes de dos estilos de actuación. Siguiendo la tradición antropológica, los nombres de los estudiantes aquí mencionados han sido cambiados para preservar su identidad.

2 Una excepción es Sáez (2017), quien sostiene que la danza jazz fue considerada, en un [continua] 
Uno de los pocos trabajos que ha buscado indagar, de manera sistemática, los límites de una cierta circulación, es el de Frigerio (2012). Frigerio se pregunta dónde se detiene el sincretismo y la reapropiación en clave new age de prácticas y creencias religiosas. A contramano de lo que ve como una tendencia académica a exaltar la inagotable capacidad sincrética del new age, Frigerio sostiene que hay ciertas religiosidades, como el pentecostalismo, la devoción por los santos populares y las religiones afroamericanas, que no suelen formar parte de este sincretismo.

En este trabajo me quiero referir a la circulación de algunos estudiantes entre estudios y escuelas de actuación privadas en la Ciudad de Buenos Aires. En particular, a la circulación durante la formación de algunos de los estudiantes que, durante un período largo, que puede durar alrededor de siete años, deciden formarse en un estilo de actuación cercano a las enseñanzas de Konstantin Stanislavski. ${ }^{3}$ Brevemente, el estilo de actuación stanislavskiano busca un tipo de "verdad escénica" similar a la de la vida cotidiana. Para ello, entrena un actor capaz de compenetrarse profundamente con su personaje y sentir en escena como este siente.

Me referiré a la circulación de estos estudiantes como el circuito realista, siguiendo a Magnani (2002), quien define a un circuito como "el ejercicio de una práctica o la oferta de determinados servicios por medio de establecimientos, y espacios que no mantienen entre sí una relación de contigüidad espacial, siendo reconocido en su conjunto por los usuarios habituales: por ejemplo, el circuito gay, el circuito de cines de arte, o el circuito neoesotérico, de los salones de danza y shows negros, del pueblo de santo, de los anticuarios, de los clubbers, entre tantos otros" (Magnani 2002: 23-24). ${ }^{4}$

Lo interesante del caso del circuito realista es que la circulación de estos estudiantes entre escuelas no equivale a una circulación que puede incluir,

cierto momento, como algo "medio grasa" entre algunas estudiantes de danza en La Plata. Sáez, además, ha señalado que no puede hablarse de un circuito de danza independiente unificado para el caso de La Plata, sino que hay circuitos diferentes con algunos puntos de conexión entre sí. Si bien la autora no ahonda en esta cuestión, esta referencia es un antecedente importante para pensar límites y lógicas particulares de circulación al interior de una misma disciplina.

3 Casi ningún estudiante de teatro circula durante toda su formación por un mismo circuito o con una misma lógica. En este artículo, me refiero a la circulación que se da durante un cierto período de esa formación, que en general comienza con un maestro que "inicia" al estudiante en una cierta visión de la actuación a la que aquel adhiere. Por falta de espacio, aquí no me refiero a ese proceso de iniciación. Utilizo el término "estilo" de un modo similar a como lo entiende Kaeppler para la danza, es decir, como "patrones persistentes que se presentan en forma de estructuras de representación - que van desde sutiles cantidades de energía al uso de diferentes partes del cuerpo - acto reconocido por las personas que pertenecen a una tradición dancística específica” (Kaeppler 2003: 103).

4 Utilizo el término "realista" dada la centralidad que el realismo como género tuvo para este estilo de actuación y, sobre todo, para evitar el término "stanislavskiano" ya que, como veremos inmediatamente, hay muchas escuelas que enseñan en este estilo en Buenos Aires pero que no forman parte de este circuito. 
de igual manera, a cualquier escuela de actuación en Buenos Aires. Tampoco se trata, a pesar de lo que podría pensarse, de una circulación delimitada por todos los estudios que enseñan en una orientación cercana a las técnicas de Stanislavski. Dicho de otro modo, no es un criterio estético o simplemente relacionado a la que sería considerada la mejor técnica el que orienta la circulación. Lo que orienta la circulación de los estudiantes del circuito realista, tal como lo pude reconstruir a partir de entrevistas y de un prolongado trabajo etnográfico es la pertenencia de los maestros que enseñan en estos estudios a un mismo linaje. ${ }^{5}$ Me refiero al linaje iniciado en Buenos Aires por Hedy Crilla (1898-1984) - de ninguna manera la única maestra que retomó las enseñanzas de Stanislavski en Buenos Aires; sin duda la más exitosa en términos pedagógicos, en su época - y sus alumnos.

En la siguiente sección describo brevemente algunos de los maestros que se interesaron y enseñaron las técnicas de Stanislavski en Argentina hacia las décadas del 50 y el 60 del siglo xx en Buenos Aires. En la tercera sección, describo brevemente el linaje y el circuito realista. En la cuarta sección, describo la historia de este linaje tal como es reconstruida por Franco, un estudiante en formación que circula por el circuito realista. Sostendré que los estudiantes de este circuito, mientras estudian actuación, también se van socializando en una cierta historia de la actuación en Buenos Aires que tiene como eje la historia del linaje que organiza el circuito y que ellos reconocen, y que puede ser entendida como una memoria colectiva en los términos de Halbwachs (1995).

\section{ALGUNAS NOTAS SOBRE LOS PRIMEROS MAESTROS QUE ENSEÑARON STANISLAVSKI EN BUENOS AIRES}

A partir de la década de 1930, y al menos hasta la década de 1960, proliferaron en Buenos Aires los teatros independientes. Inspirados en una corriente europea similar, se trataba de grupos que se oponían al teatro profesional - así se denominaba al teatro en el que los actores cobraban por su trabajo -, al que veían degradado por su mercantilización. Sus integrantes no cobrarían por su trabajo, sino que se dedicarían a la actuación después de su horario laboral. Era en este sentido que eran independientes: las obras elegidas por estos teatros no especulaban con un éxito de taquilla, sino que buscaban mostrar al público local un repertorio renovado, de vanguardia, acorde a lo que sucedía en Europa en ese momento (Risetti 2004).

Los teatros independientes tuvieron originalmente un objetivo político y pedagógico, y no comenzaron a interesarse por la formación de sus actores 
hasta la década de 1950. Es en esta época que empiezan a aparecer algunos importantes maestros, la mayoría de ellos provenientes de - o habiendo viajado a - Europa, interesados por la formación de los actores. Quisiera mencionar brevemente a algunos de estos maestros.

Obarrio (1998) señala que la creación del teatro Fray Mocho, en 1951, se dio como una escisión en el teatro independiente La Máscara, por un grupo que quería crear una escuela en el teatro y volver la asistencia a sus clases obligatoria para sus miembros. Este grupo era liderado por Oscar Ferrigno - que había estudiado en el Conservatorio de Música y Declamación y había pasado dos años formándose en Francia con Jacques Copeau -, que luego sería el principal referente del teatro Fray Mocho. El teatro Fray Mocho tuvo su propia escuela, algo nada común en la época, ya que la formación no estaba entre las preocupaciones centrales de los teatros independientes. La escuela del Fray Mocho contaba con materias prácticas como gimnasia para actores, expresión corporal, técnica vocal y prácticas de improvisación. A su vez, tuvo su propia publicación, los Cuadernos de Arte Dramático, entre 1951 y 1954. El número 6 estuvo dedicado a Stanislavski, a quien los estudiantes de Fray Mocho ya venían investigando, junto a otras técnicas en las que Ferrigno se había formado en Francia, bajo la influencia de Jaques Copeau.

En 1948, Galina Tolmacheva, actriz rusa nacida en 1895 y llegada a Argentina en 1925, crea la Escuela de Teatro de Mendoza, dependiente de la Universidad de Cuyo. El estudio de las artes dramáticas comprendía tres años e incluía materias de arte dramático, técnica de la voz, técnica del movimiento e historia del teatro. Tolmacheva escribió y publicó Creadores del Teatro Moderno, libro en el que hablaba sobre algunos maestros europeos de la época, hasta entonces relativamente desconocidos en el país. Entre ellos se encontraba Stanislavski. Esta pedagoga es reconocida por haber sido una de las primeras en implementar sus enseñanzas en Argentina (Cortese 1998).

Otra figura insoslayable de la época es la de Oscar Fessler. Fessler nació en 1914 en Batra Dornei, un pueblo que formaba parte del Imperio Austrohúngaro. Con el ascenso de Hitler, Fessler, de origen judío, huyó primero a Dinamarca, y luego se instaló en París, en donde estudió con Alexis Granovcki, discípulo directo de Stanislavski. Fessler también había tenido entre sus maestros a Max Reinhardt y Erwin Piscator, y conocía bien el trabajo de Brecht. Llegó a Argentina en 1957, invitado por el Idisher Folks Theater (IFT) a dirigir El Triste Fin de Amán (Tríbulo 2014; Paolantonio y Degregori 2017). Fessler estuvo al frente de la Escuela Provincial de Teatro de Santa Fe, entre 1959 y 1962, y del Instituto de Teatro de la Universidad de Buenos Aires (ITUBA), dependiente del área de extensión de esta universidad, entre 1959 y 1966. También fue docente en el ITUBA Juan Carlos Gené, otro importante maestro de actores de la época que se interesaría por las enseñanzas de Stanislavski. 
Por otra parte, Agustín Alezzo y Augusto Fernandes venían de integrar el Nuevo Teatro, un teatro independiente dirigido por Pedro Asquini y Alejandra Boero. En 1955 se separaron de este teatro formando el grupo Juan Cristóbal, que en 1957 se integró al teatro independiente La Máscara. En 1959, Alezzo, Fernandes y otros miembros de La Máscara buscaron un maestro con quien formarse y mejorar su actuación. Fernandes cuenta que eran considerados por sus colegas los pequeño-burgueses que querían formarse y estudiar (Risetti 2004), lo que da cuenta de que, si bien había en el teatro independiente un cierto interés por la formación, esta continuaba siendo polémica y no formaba parte del sentido común de la época.

Este grupo comenzó a estudiar con la actriz austríaca Hedy Crilla. Crilla había huido de Europa durante el nazismo y se encontraba actuando - y también enseñando - en Buenos Aires hacía casi 20 años, en compañías alemanas y francesas, y en obras infantiles. Después de un breve período en el que estos actores aprendieran en su estudio, Crilla comenzó a enseñarles en el sótano del teatro La Máscara.

Si bien Stanislavski ya venía siendo estudiado e investigado en diferentes grupos desde hacía algunos años, el estreno de Cándida de Bernard Shaw, en 1959, con dirección de Hedy Crilla y Carlos Gandolfo (encargándose ella de la dirección de actores y él de la puesta en escena), fue la gran primera muestra de cómo las enseñanzas del maestro ruso podían producir actuaciones novedosas. Varios actores o estudiantes de actuación de la época - como Norma Aleandro o Pino Solanas - cuentan que quedaron fascinados con las actuaciones de Cándida, así como con Una Ardiente Noche de Verano de Ted Williams y con Espectros de Henrik Ibsen, las dos puestas siguientes dirigidas por Crilla con actores de La Máscara (Roca 1998). La obra consagró a Crilla como maestra de actores, y convirtió sus talleres en una referencia central para el estudio de actuación en Buenos Aires en los años que siguieron.

\section{EL CIRCUITO REALISTA HOY}

El estilo de actuación stanislavskiano ocupó un lugar totalmente central durante las décadas del 60 y el 70 y muchos maestros de actuación lo experimentaron de una u otra manera. En algunos casos, estos maestros se habían formado con otros maestros que ya habían experimentado con estas enseñanzas; en otros, las abordaron directamente a partir de los libros.

A partir de la década del 80 del siglo xx y con la vuelta de la democracia, otros tipos de estilos de actuación comenzaron a proliferar en Buenos Aires (entre muchos otros, el clown, la técnica Lecoq y la antropología teatral).

Paralelamente, los maestros del estilo stanislavskiano siguieron enseñando. También, muchos de sus alumnos se convirtieron en maestros y comenzaron a enseñar en este estilo. 
Entre los estudiantes de actuación, algunos acaban formándose durante largos períodos con maestros que enseñan en este estilo. Sin embargo, entre aquellos que entrevisté y otros que conocí o cuya historia investigué durante el período en que realicé mi trabajo de campo (del 2014 al presente), quedó claro que estos estudiantes no se forman con cualquier maestro que enseñe este estilo de actuación, sino en ciertos estudios que forman parte de lo que aquí llamo el circuito realista.

Me refiero al circuito realista como la circulación de estudiantes entre los maestros que originalmente se formaron con Hedy Crilla en las enseñanzas de Stanislavski - Augusto Fernandes (n. 1939), Agustín Alezzo (n. 1935) y Carlos Gandolfo (1931-2005) -, así como entre algunos ex alumnos de estos maestros devenidos ellos también maestros. Como hemos visto, hubo otros maestros que también se formaron en y enseñaron las técnicas de Stanislavski, y que a su vez tuvieron alumnos que luego devinieron maestros. Sin embargo, la circulación de los estudiantes de este circuito suele limitarse a los maestros que lo enseñan al interior de este linaje, y no a todos los maestros que enseñan un estilo de actuación stanislavskiano. Permítanme describir brevemente a algunos de los maestros de este circuito.

Agustín Alezzo dirige la escuela El Duende desde 1966. ${ }^{6}$ La escuela propone una formación de cuatro años para actores. Alezzo da clases en el cuarto año - con anterioridad también daba el tercer año -, así como un seminario para actores formados al que pueden acceder ex alumnos de la escuela y otros estudiantes formados en otras escuelas - luego de pasar una prueba de admisión -. Por su parte, luego de una larga estadía en Alemania durante la década del 70, Augusto Fernandes comienza a dar cursos en Buenos Aires de forma intermitente durante la década del 80 y luego regularmente a partir de la década del 90. En su caso, su estudio no se encuentra diferenciado en años, sino que hay diferentes grupos, diferenciados por su experiencia.

De una generación más joven, Julio Chávez (n. 1956) también aparece como un maestro central de este circuito. Chávez es un caso paradigmático de un estudiante - luego devenido maestro - que se forma circulando entre los maestros del circuito realista. Chávez estudió con Agustoni, con Alezzo, con Gandolfo y con Fernandes. Por sus éxitos en televisión y en cine, y por la continuidad de su trabajo en teatro - primero como actor, luego como maestro

6 La mayoría de las escuelas de los maestros del circuito realista consisten en un programa curricular de cuatro años en los que se van enseñando diferentes habilidades y se van realizando obras de diferentes autores. En general, el maestro que es referente de la escuela enseña en el último año, así como en un seminario actual para actores al que pueden acceder quienes han terminado la formación básica de cuatro años, así como estudiantes que ya poseen una formación similar, en otra u otras escuelas. El estudio de Augusto Fernandes es una excepción en este sentido, ya que este maestro sólo enseña en talleres que no siguen una formación preestablecida y en la que se debe ir pasando de un año a otro. 
y director -, ocupa un lugar central en el circuito, en tanto los estudiantes que lo recorren estudian con él y lo mencionan de manera recurrente.

El circuito realista que aquí vengo describiendo se basa en entrevistas realizadas a estudiantes entre 2016 y 2017 , y en este sentido es una "foto", es decir muestra un momento específico de este circuito. ${ }^{7}$

El esquema de la figura I muestra a los maestros de este linaje - el iniciado por Hedy Crilla y sus alumnos - que se encentraban dando clases durante mi trabajo de campo. Como se observa, Hedy Crilla y Carlos Gandolfo están ausentes del esquema, debido a que habían fallecido con anterioridad. Los maestros que se encuentran al interior del círculo central son los más renombrados del linaje, es decir, aquellos con los que, en la época, se estudiaba con mayor frecuencia. En cuanto a los que se encuentran afuera del círculo, el esquema sólo muestra a aquellos que fueron mencionados con mayor frecuencia por mis entrevistados.

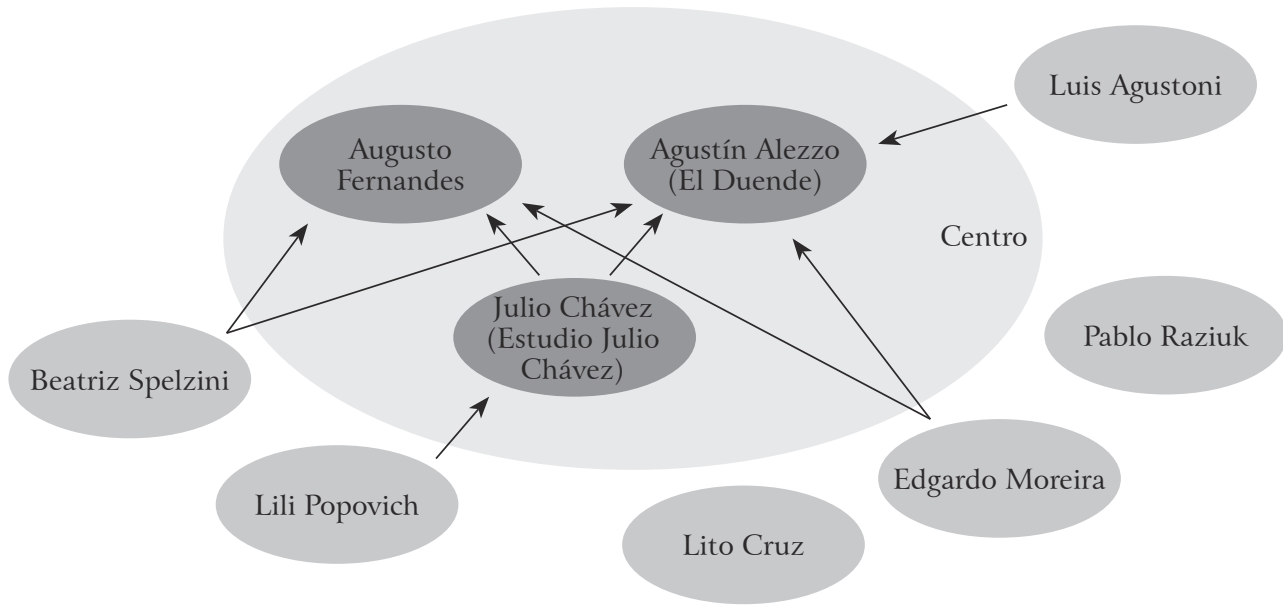

Figura 1 - Los maestros activos en Buenos Aires del linaje realista hacia 2016-2017 y los principales maestros con quien se formaran.

7 Las entrevistas se realizaron en estos años y se trataba de estudiantes que aún estaban circulando por el circuito o todavía circulaban en los años anteriores, a lo sumo dos o tres años antes de la entrevista. 


\section{FRANCO: "LO QUE TE DIGO ES LO QUE ÉL ME CONTÓ”}

De acuerdo a lo que sostienen en entrevistas y conversaciones personales, la gran mayoría de los estudiantes que recorren el circuito realista no aprenden la historia del teatro o de la actuación en Buenos Aires a partir de libros o de clases de historia del teatro. Por el contrario, esa historia se va reconstruyendo de manera errática, a partir de fragmentos que escuchan de sus maestros o de otros compañeros. Estas historias pueden ser entendidas como memorias colectivas en los términos de Halbwachs, quien sostiene que la memoria colectiva se diferencia de la historia en tanto es una corriente de pensamiento "que no tiene nada de artificial, puesto que retiene del pasado sólo lo que aún está vivo o es capaz de vivir en la conciencia del grupo que la mantiene. Por definición, no excede los límites del grupo" (Halbwachs 1995: 213) y, dado que en cualquier sociedad existen varios grupos, existen, también, diferentes memorias colectivas. Veamos cómo Franco cuenta el momento fundacional del linaje realista.

Cuando terminó la escuela, Franco, que nació en Buenos Aires en 1988, trató de entrar al IUNA y a la EMAD pero le fue mal en los dos. ${ }^{8}$ Su madre le imprimió un listado de maestros; cuando vio el nombre de Alezzo decidió que quería estudiar con él, porque recordaba haber leído en una nota en el diario que Alezzo había sido el maestro de Alfredo Alcón, uno de sus actores preferidos en ese entonces. Estudió ocho años en El Duende, los primeros dos con Lisardo Laiphitz y los siguientes seis con Alezzo. Franco también actuó en algunas obras de Alezzo y actualmente da clases en el primer año de su escuela.

Como otros estudiantes con los que hablé que se formaron en el circuito realista, Franco cuenta el momento en que, hacia 1959, Alezzo, Gandolfo y Fernandes fueron a buscar a Hedy Crilla para que les enseñara a actuar en las enseñanzas de Stanislavski como uno de los momentos fundacionales del teatro argentino:

“Toda esa historia es fascinante sobre todo por la determinación que tuvieron ellos, y cambiaron todo el teatro argentino contemporáneo. Si vos comparás el teatro argentino, o los actores más que el teatro, acá en Argentina con el resto de Latinoamérica, no tiene nada que ver, es otra cosa. A mí me parece que tenemos una calidad de actores increíble, que creo que parte de ahí, parte de ahí, de esa obstinación que tenían ellos que, él [Alezzo] me cuenta que '¿qué pasaba?, a nosotros nos gustaba el teatro pero no nos gustaba el teatro que veíamos acá, veíamos las películas de todos los actores del Actor's Studio, las películas de Elia Kasan con toda esa camada, con Pacino,

8 La EMAD es la Escuela Metropolitana de Arte Dramático y el IUNA el Instituto Universitario Nacional de las Artes, actualmente UNA (Universidad Nacional de las Artes). 
no sé si tanto Pacino, con Brando, con James Dean. Nosotros veíamos y decíamos esto es lo que queremos'. Esa determinación es maravillosa, eran pibes de 17, 18 años, que veían teatro acá y decían, me gusta el teatro pero esta actuación no me gusta, e iban a ver cine y decían 'esto, esto, esto queremos’ y empezaron a buscar. Y se juntaron él [Alezzo], Gandolfo, Fernandes y unos cuantos más, y empezaron a buscar profesores, eso es rarísimo. Por lo general el alumno va a tomar clases con el profesor. Ellos no, ellos traían profesores y los echaban, 'No, no, esto no es lo que queremos'. Estaban con otro un mes, ‘no, no, esto no es lo que queremos'. Hasta que dieron con Hedy Crilla. Y con la Crilla, dice [Alezzo] que tuvieron una clase y dijeron 'sí, esto, esto, esto es lo que queremos'. Esa convicción es, yo no sé, a mí me fascina. Cambiaron la historia del teatro argentino, por una obstinación que tenían que, no sé, que les estaba predestinada, no sé, hay algo de esa obstinación que me parece fantástico. Mirá, estos tipos venían a cambiar el teatro argentino y estaban empecinados en que querían algo particular, y querían algo, querían algo, y después fueron los tres maestros que fueron, y Alezzo es el día de hoy que todo el medio lo respeta de una manera increíble. El otro día, cada vez que pasa a recibir un premio o a dar un premio, le dan unos aplausos que se te caen las medias, y que él mismo se emociona mucho, y él me dice, 'yo no sé por qué me aplauden tanto, está bien yo trabajé mucho, y siempre con ganas, con energía y con una idea de trabajo que siempre respeté y nunca traicioné, pero a mí me sorprende el amor que me demuestra la gente de teatro'. Y yo creo que tiene que ver con el entendimiento de esto, de... mirá, estos chabones, a fuerza de trabajo, a fuerza de una idea clara, dieron vuelta el teatro argentino" [Franco, 27 años, entrevista realizada en noviembre de 2016].

Para Franco, y para la mayoría de los que, como él, se forman entre los maestros del circuito realista, el momento en que Alezzo, Gandolfo y Fernandes fueron a buscar a Hedy Crilla es un momento fundacional, hay un antes y un después en el teatro argentino. Volviendo sobre su historia, Franco me cuenta que con el tiempo descubrió que su llegada a la escuela de Alezzo había sido un error, y que Alezzo nunca había sido el maestro de Alcón, aunque lo había dirigido algunas veces en el teatro, de allí la confusión. Hoy por hoy, Alcón no es el tipo de actor que Franco quiere ser. "Alcón se formó en otra rama, viene de una escuela más declamatoria, que es anterior a esta búsqueda que después hacen Alezzo y los otros, que busca trabajar con las emociones y la verdad en escena”, me explica Franco. ${ }^{9}$ La declamación o la escuela declamatoria es, en la

9 En las clases de este estilo de actuación stanislavskiano se suele dedicar bastante tiempo a componer escenas tomadas de obras de teatro. Tal como enseñan los maestros, los estudiantes tienden a partir del análisis de la obra de teatro para comprender el personaje que van a componer. [continua] 
historia de Franco y para otros que recorren el circuito realista, aquella forma de actuación que imperaba en el teatro argentino con anterioridad, y que la llegada de sus maestros vendría a cambiar por un teatro que busca poner la "verdad en escena".

La reconstrucción de una historia del teatro al interior de cada circuito - una memoria colectiva en los términos de Halbwachs - comienza en la clase misma. De a poco, en un proceso que no tengo lugar para describir acá, el alumno va descubriendo cuáles son los actores y los directores que más interesan a su maestro, así como las relaciones que existen entre ellos. También descubre con quién estudió su maestro, cuál es la tradición a la que él adhiere, qué otros actores y directores se encuentran en una línea similar. Así, la reconstrucción de la historia de la actuación porteña al interior de cada circuito comienza por una reconstrucción del linaje en que el maestro se inscribe, y asimismo del lugar - central, para quien está en el circuito - que esa tradición ocupa en la historia reciente del teatro. El modo en que esta historia se reconstruye jerarquiza a algunos maestros, así como, en alguna medida, a todo aquel que se inscriba en ese linaje, y da cuenta, al menos en parte, del hecho de que la circulación de los estudiantes que se forman en el circuito realista se dé sólo entre maestros del linaje, y no con cualquier maestro que enseña en el estilo stanislavskiano.

De este modo, el maestro del maestro suele ser la primera alternativa del estudiante, cuando decide estudiar en otro estudio, y esto se debe a las innumerables menciones positivas que su maestro ha hecho, a su vez, de quien lo formó a él. Así, luego de haber estudiado con un maestro que lo ha cautivado profundamente - y con el que ha aprendido una sensibilidad que es la del linaje en el que su maestro está inscrito -, el estudiante continúa circulando entre maestros que, no sólo enseñan el mismo estilo de actuación, sino que también pertenecen al mismo linaje. Como acabo de mencionar, lo más común es que los estudiantes continúen su camino yendo a estudiar con alguno de los maestros de su maestro. En algunos casos, esto se da naturalmente al interior de una escuela, cuando un estudiante pasa de estudiar con alguno de los maestros que dan las clases para principiantes - y recién llegados - a estudiar con el maestro principal de ese estudio. En otros casos, ese desplazamiento es parte de una migración hacia la Ciudad de Buenos Aires, donde enseña el maestro del maestro. Esto se debe a que algunos maestros enseñan en ciudades de la Provincia de Buenos Aires o en el interior de la Argentina. En algunos casos también, los estudiantes no se mudan, sino que viajan semanalmente a Buenos Aires para estudiar con el maestro de su maestro, algo que

A continuación, desarrollan ciertas estrategias para entrar en el "estado" emocional que les permita actuar de un modo acorde al personaje que tienen que interpretar y generar esa "verdad en escena" a la que se refiere Franco. 
sucede desde ciudades como La Plata (Del Mármol 2016), que se encuentra a $60 \mathrm{~km}$ de la Capital Federal, y Rosario, que se encuentra a $300 \mathrm{~km} .{ }^{10}$ Por último, algunos estudiantes cambian de estudio, y van a estudiar a la escuela del maestro de su maestro.

Finalmente, quisiera enfatizar que el modo en que se reconstruye la historia de la actuación porteña es muy diferente para estudiantes de actuación con diferentes trayectorias. Para un estudiante que no se ha formado con maestros del circuito realista, lo más probable es que el nombre Hedy Crilla no signifique absolutamente nada, de hecho, nunca lo hayan escuchado nombrar. Quisiera mencionar como ejemplo el caso de los estudiantes que se forman entre estudios del teatro de estados, otro circuito al que he seguido como parte de mi investigación etnográfica. La historia de la actuación porteña que se reconstruye entre estos estudiantes es sumamente diferente. Nuevamente en ese caso, el origen de ese linaje - marcado por directores como Alberto Ure y Ricardo Bartís -, presenta un antes y un después en la historia de la actuación porteña. Ese "antes", lo que estaba con anterioridad, lo "viejo", no es ya la declamación, como en la historia de Franco y de los estudiantes del circuito realista, sino las actuaciones basadas en las técnicas de Stanislavski. A su vez, para los actores del circuito del teatro de estados, el estreno de Postales Argentinas, en 1989, una de las primeras obras dirigidas por Bartís y escrita junto a Pompeyo Audivert, y en la que este también actuaba, aparece como una referencia fundamental, un marco de un antes y un después en el teatro argentino. Este momento presenta dos antecedentes fundamentales: el trabajo de Alberto Ure - que suele ser referido a través de un libro que recopila varios de sus ensayos, Sacate la Careta -, y el under, término utilizado para hablar de un cierto teatro que renovó la escena porteña durante la década de los 80 .

\section{CONCLUSIONES}

Los trabajos de Magnani fueron probablemente, a través de la noción de circuito, los que más han contribuido a pensar los modos en que ciertos grupos recorren la ciudad. Aquello que estos grupos tienen en común, suelen ser criterios estéticos, éticos, identitários o simplemente, gustos en común.

Como he querido mostrar aquí, en el caso de los estudiantes de actuación que circulan por el linaje realista, aquello que orienta su circulación entre maestros en el contexto de su formación no es la simple pertenencia a un estilo

10 La relación entre migración e ir a estudiar con el maestro de su maestro también fue apuntada por Downey (2010) y Dalidowicz (2012) - ver también Downey, Dalidowicz y Mason (2015) - para los casos del aprendizaje del capoeira y la danza kathak, quienes, al decidir continuar su trabajo de campo etnográfico en un país diferente del que lo habían comenzado, cuentan que fueron a estudiar con los maestros de sus maestros - en el caso de Downey, en Nueva York, y en el de Dalidowicz, en Calcuta. 
de actuación al que ellos adhieren o a una estética. A lo largo de su formación, y de manera paralela a que se van formando en una cierta sensibilidad y van desarrollando una preferencia por un estilo de actuación - como he mostrado en otra parte (Battezzati 2017) -, los estudiantes que se forman entre los maestros del linaje realista van siendo socializados en una memoria colectiva que tiene como eje la historia de ese linaje. Es a partir de allí que empiezan a desarrollar una visión de la historia de la actuación de su país. La cercanía a los grandes maestros y a los grandes hitos de la historia de ese linaje es lo que otorga una cierta jerarquía a unos maestros por sobre otros, orientando un tipo de circulación que no puede ser reducida a la simple adherencia a un estilo particular.

\section{BIBLIOGRAFÍA}

BATTEZZATI, Santiago, 2017, Histriónicos y Emocionales: La Formación de los Actores en Dos Estilos de Actuación en Buenos Aires. San Martín, Universidad de San Martín, tesis de doctorado en Antropología.

CAROzZI, María Julia, 2000, Nueva Era y Terapias Alternativas: Construyendo Significados en el Discurso y la Interacción. Buenos Aires, EDUCA.

CAROZZI, María Julia, 2015, Aquí se Baila el Tango: Una Etnografía de las Milongas Porteñas. Buenos Aires, Siglo XXI.

CORTESE, Nina, 1998, Galina Tolmacheva o el Teatro Transfigurado. Buenos Aires, Instituto Nacional del Teatro.

DALIDOWICZ, Monica, 2012, Learning Kathak: Crafting Bodies and Selves in the Guru Shishya Parampara. Sydney, Macquarie University, tesis de doctorado en Antropología.

DEL MÁRMOL, Mariana, 2016, Una Corporalidad Expandida: Cuerpo y Afectividad en la Formación de los Actores y Actrices en el Circuito Teatral Independiente de la Ciudad de La Plata. Buenos Aires, Universidad de Buenos Aires, tesis doctoral.

DOWNEY, Greg, 2010, “'Practice without theory': a neuroanthropological perspective on embodied learning”, Journal of the Royal Anthropological Institute, 16 (1): 22-40.

DOWNEY, Greg, Monica DALIDOWICZ, y Paul H. MASON, 2015, "Apprenticeship as method: embodied learning in ethnographic practice", Qualitative Research, 15 (2): 183-200.

FRIGERIO, Alejandro, 2012, "Lógicas y límites de la apropiación new age: donde se detiene el sincretismo”, en R. De la Torre, C. G. Zúñiga y N. Juárez Huet (comps.), Variaciones y Apropiaciones Latinoamericanas del New Age. México, Centro de Investigaciones y Estudios Superiores en Antropología Social, El Colegio De Jalisco.

HALBWACHS, Maurice, 1995, "Memoria colectiva y memoria histórica", REIS: Revista Española de Investigaciones Sociológicas, 69: 209-219.

Infantino, Julieta, 2014, Circo en Buenos Aires: Cultura, Jóvenes y Políticas en Disputa. Buenos Aires, Instituto Nacional del Teatro. 
KAEPPLER, Adrienne Lois, 2003, "La danza y el concepto de estilo", Desacatos, 12: 93-104. MAGNANI, José Guilherme Cantor, 1999, "O circuito neo-esotérico na cidade de São Paulo”, en M. J. Carozzi (comp.), A Nova Era no Mercosul. Petrópolis, Vozes, 27-46.

MAGNANI, José Guilherme Cantor, 2002, "De perto e de dentro: notas para uma etnografia urbana”, Revista Brasileira de Ciencias Sociais, 17 (49): 11-29.

OBARRIO, Estela, 1998, Teatro Fray Mocho 1950-1962: Historia de Una Quimera Emprendida. Buenos Aires, Instituto Nacional del Teatro.

OSSWALD, Denise, 2015, "Deshacer los hábitos bailando: concepciones alternativas del cuerpo en la transmisión dancística independiente en Buenos Aires”, en M. J. Carozzi (comp.), Escribir las Danzas: Coreografías de las Ciencias Sociales. La Plata, Editorial Gorla, 207-237.

PAOLANTONIO, José María, y Oscar Roque DEGREGORI, 2017, El Irreverente Mensaje del Teatro: Oscar Fessler en Santa Fe. Buenos Aires, edición de autor.

RAHAIM, Matthew, 2012, Musicking Bodies: Gesture and Voice in Hinduistani Music. Middletown, Wesleyan University Press.

RISETTI, Ricardo, 2004, Memorias del Teatro Independiente Argentino, 1930-1970. Buenos Aires, Corregidor.

ROCA, Cora, 1998, Hedy Crilla: La Palabra en Acción. Buenos Aires, Instituto Nacional del Teatro.

SÁEZ, Mariana Lucía, 2017, Presencias, Riesgos e Intensidades: Un Abordaje Socio-Antropológico sobre y desde el Cuerpo en los Procesos de Formación de Acróbatas y Bailarines/as de Danza Contemporánea en la Ciudad de La Plata. Buenos Aires, Universidad de Buenos Aires, tesis de doctorado.

Tríbulo, Juan (comp.), 2014, El Pensamiento Vivo de Oscar Fessler. Tomo 1: El Juego Teatral en la Educación. Buenos Aires, Instituto Nacional del Teatro. 